\title{
Impact of bead-beating intensity on microbiome recovery in mouse and human stool: Optimization of DNA extraction
}

Bo Zhang1 ${ }^{*}$, Matthew Brock ${ }^{*}$, Carlos Arana ${ }^{1}$, Chaitanya Dende ${ }^{1}$, Lora Hooper $^{1}$ and Prithvi Raj ${ }^{*}$

${ }^{1}$ Department of Immunology, University of Texas Southwestern Medical Center, Dallas, TX 75390, USA *Microbiome Research Laboratory (MRL)

\section{Correspondence:}

Prithvi Raj, Microbiome Research Laboratory (MRL) Department of Immunology, University of Texas Southwestern Medical Center, Dallas, TX 75390, USA Email:

prithvi.raj@utsouthwestern.edu

Keywords: Microbiome, 16S Sequencing, OTU, DNA extraction, Bead beating 


\section{Abstract}

2 DNA extraction methods play an important role in the acquisition of accurate and reproducible

3 16S sequencing data in microbiome studies. In this study, we assessed the impact of bead-beating

4 intensity during DNA extraction on microbiome recovery in mouse and human stool. We observed

5 a higher DNA yield, better DNA integrity, higher Shannon's entropy and Simpson's index in

6 samples beaten for 4 and 9 minutes as compared to unbeaten samples. 16S sequencing data showed

7 that bead beating has a statistically-significant $(\mathrm{p}<0.05)$ impact on the recovery of many clinically

8 relevant microbes that live in the mouse and human gut, including Bifidobacterium, Sutterella and

9 Veillonella. It was observed that 4 minutes of bead beating promotes recovery of about $70 \%$ of

10 OTUs in mouse and human stool, while the remaining 30\% requires longer bead beating. In

11 conclusion, our study indicates adjustments in bead beating treatment based on the composition of

12 the specimen and the targeted bacteria.

13

14

15

16

17

18 


\section{Introduction:}

25 High throughput sequencing technology is commonly used to characterize microbial composition

26 of biological specimens. This approach can be applied to capture microbial diversity in human and

27 environmental specimens with unprecedented depth (1-4). A number of prior studies provide

28 evidence that methods of sample collection, storage and DNA extraction are critical for accurate

29 profiling of microbiota in environmental (5-7) or human samples (8-10). In particular, it is

30 increasingly apparent that the DNA extraction method is crucial to the accuracy of microbiome

31 analysis (11-13). Given that the microbial composition of a niche is generally diverse with

32 significant variations in cell membrane structures and functions among community members,

33 obtaining a complete and unbiased representation of microbial DNA from all community members

34 is technically challenging.

36 There is growing evidence that complete lysis of bacterial cell walls is critical for optimum yield

37 of DNA. Lysis protocols include procedures that lead to physical and or enzymatic disruption of

38 the microbial cell wall $(5,14,15)$. It has been observed that extended lysis time and mechanical

39 disruption can enhance nucleic acid yield. However, extended lysis time can also reduce molecular

40 complexity by shearing genomic DNA into smaller fragments $(16,17)$. In general, bacterial cells

41 are lysed to release the nucleic acids and the remaining proteins are discarded. Gram-positive

42 bacteria pose the greatest challenge for complete lysis due to their thick cell walls and complex

43 cell wall composition, consisting of several layers of peptidoglycan (18).

45 Given that the precise composition of pathogenic clinical specimens is mostly unknown and may 46 vary significantly from sample to sample, an ideal DNA extraction method should accurately 
47 recover DNA from a wide variety of bacteria and avoid the bias that can be introduced by

48 incomplete cell wall lysis. Bead-beating is a method of mechanical disruption that is performed

49 prior to standard DNA extraction. In this step, ceramic or glass beads are added to the tube

50 containing microbial samples. This is followed by moderate to high speed shaking, causing

51 collisions between the beads and the samples. Bead-beating has become a common method of

52 bacterial cell lysis in microbial metagenomics studies, and a number of different bead beating

53 protocols have been used to extract microbial DNA from stool samples (19). Here we have

54 assessed the impact of bead-beating time on extraction efficiency of nucleic acids and abundance

55 and composition of bacterial OTUs in mouse and human stool.

Materials \& Methods

\section{Sample collection}

60 We collected two mouse (C57/B16) stool samples, designated WT1 and WT2, and two human

61 stool samples, designated Hum1 and Hum2. The stool samples were collected under sterile

62 conditions and stored in DNA/RNA shield, a nucleic acid stabilizing solution from Zymo Research

63 (R1100). DNA/RNA shield provides an accurate molecular signature of the sample at the time of

64 collection by preserving nucleic acids at ambient temperature and inactivating organisms including 65 infectious agents.

\section{DNA extraction}

68 We used the ZymoBIOMICS ${ }^{\text {TM }}$ DNA Miniprep Kit (D4300) for DNA extraction on both mice and

69 human stools. Figure 1 illustrates the experimental workflow of the study. Each of the mouse and 
70 human stool samples was aliquoted into four subsamples for the experiment. About $200 \mathrm{mg}$ of

71 feces was aliquoted into a ZR BashingBead lysis tube $(0.1$ and $0.5 \mathrm{~mm})$. For lysis, $750 \mathrm{ul}$ of

72 ZymoBIOMICS lysis solution was added to each sample tube. Next, each sample tube was tightly

73 closed and loaded onto the PowerLyzer 24 Homogenizer (110/220 V) from Qiagen for bead

74 beating. WT1 and WT2 and Hum1 and Hum2 were two independent replicates of mouse and

75 human feces, respectively. We selected four different bead beating time points as illustrated in

76 Figure 1: 0 minutes (no bead-beating at all), 1 minute (one cycle of shaking), 4 minutes (2 cycles

77 of 2 minute shaking, with a 30 second pause after each cycle) and 9 minutes (4 cycles of 2 min

78 and 1 cycle of 1 minute, with a 30 second pause after each cycle). Each of these samples were

79 bead-beaten at a speed of 2200 RPM and were maintained at a temperature of $20^{\circ} \mathrm{C}$ throughout the

80 bead beating process. Following beat-beating and lysis, DNA was purified using the

81 ZymoBIOMICS protocol, and 100 ul was eluted for downstream experiments. The DNA

82 concentration was measured using the Picogreen method (Invitrogen Quant-iT ${ }^{\mathrm{TM}}$ Picogreen

83 dsDNA Assay Kit Reference No. P11496 on Perkin Elmer 2030 Multilabel Reader Victor X3) and

84 DNA integrity number (DIN) was determined on 4150 Tapestation from Agilent using Agilent's

85 gDNA Screen Tape (Reference No. 5067-5365) and Agilent's gDNA Reagents (Reference No.

86 5067-5366).

$88 \quad 16 S$ rRNA gene sequencing

$89 \quad 10-50 \mathrm{ng}$ of purified DNA from stool was used to amplify hypervariable region V3-V4 of the

90 bacterial 16S rRNA gene using the Illumina Nextera protocol (Part \# 15044223 Rev. B). A single

91 amplicon of about 460 bp was amplified using the 16S Forward Primer

92 (5'TCGTCGGCAGCGTCAGATGTGTATAAGAGACAGCCTACGGGNGGCWGCAG) and 
94 (5'GTCTCGTGGGCTCGGAGATGTGTATAAGAGACAGGACTACHVGGGTATCTAATCC

95 ) as described in the Illumina protocol. The PCR product was purified using Agencourt AmpureXP

96 beads from Beckman Counter Genomics. We used the Nextera XT Index Kit v2 (Reference no.

97 15052166) for $16 \mathrm{~S}$ amplification. Illumina adapter and barcode sequences were ligated to the

98 amplicon in order to attach them to the MiSeqDx flow cell and for multiplexing. Quality and

99 quantity of each sequencing library were assessed using Bioanlyzer and picogreen measurements,

100 respectively. The libraries were then pooled in equal concentrations according to picogreen

101 measurements. Each pool was quantified using KAPA Biosystems Library Quant Kit (illumina)

102 ROX Low qPCR Mix (Reference No. 07960336001) on an Applied Biosystems 7500 Fast Real-

103 Time PCR system. According to the qPCR measurements, $6 \mathrm{pM}$ of pooled libraries was loaded

104 onto a MiSeqDX flow cell and sequenced using MiSeq Reagent Kit v3 600 Cycles PE (Paired end

$105300 \mathrm{bp}$ ). Raw fastq files were demultiplexed based on unique barcodes and assessed for quality.

106

$107 \quad$ 16S data analysis pipeline

108 Samples with more than 50K QC pass sequencing reads were used for downstream 16S OTU 109 analysis. Taxonomic classification and Operational Taxonomic Units (OTUs) abundance analysis 110 were done using the CLC Bio microbial genomics module

111 (https://www.qiagenbioinformatics.com/plugins/clc-microbial-genomics-module/). Individual

112 sample reads were annotated with the Greengene database and taxonomic features were identified.

113 Alpha and beta diversity analysis was done to understand within- and between-treatment group

114 diversity, respectively. Raw fastq files from this study have been submitted to the Sequence Read

115 Archive with ID PRJNA625828. 


\section{Results}

\section{Assessment of DNAs extracted using different bead beating times}

119 First, we measured the amount of total DNA recovered from each bead-beating treatment. As

120 expected, the bead-beaten samples yielded higher amounts of DNA as compared to unbeaten

121 samples. As shown in Supplementary Fig.1 A-B, the highest yields were observed in samples

122 beaten for 4 or 9 minutes. The DNA integrity number (DIN) was highest in samples treated for 1

123 and 4 minutes (Supplementary Fig.1C-D). The number of pass filter sequencing reads was highest

124 in mouse stool samples that were beaten for 4 and 9 minutes (Supplementary Fig.1E). However,

125 in human stool samples, the highest pass filter reads were obtained at the 1 and 4-minute time

126 points (Supplementary Fig.1F). We also compared the total number of high-confidence OTUs

127 annotated in all the samples. As shown, the highest OTUs were observed in samples beaten for 4

128 or 9 minutes (Supplementary Fig. 1G-H). Overall, 4 minutes of beating time was found to give the

129 optimum results for all the assessed parameters.

\section{Actinobacteria requires extensive bead beating for maximal recovery}

132 QC pass sequencing reads were used to define OTUs (operational taxonomic units) at different

133 taxonomic levels such as phylum, class, order, family, genus, and species (Fig. 2A-B,

134 Supplementary Table S1-S4). 16S analysis showed that Actinobacteria were significantly $(\mathrm{p}<0.05)$

135 underrepresented in unbeaten samples. Their maximal recovery was observed after 4 and 9-

136 minutes of bead-beating (Fig.2C-D). On the other hand, Proteobacteria, which are Gram-negative

137 organisms, were better captured in unbeaten samples or after just 1 minute of bead beating (Fig.

138 2I\&J). Bacteroidetes were least affected by bead-beating time in both mouse and human stool

139 samples (Fig. 2G\&H). Results for Firmicutes were not consistent between mouse and human 
140 samples, as more Firmicutes were recovered at 4 and 9 minutes of bead-beating of mouse stool

141 whereas no such trend was observed in the human samples. The aggregated phylum level

142 abundances and comparative statistics between time points in mouse and human data are given in

143 Supplementary Tables S3 and S4, respectively. Differential abundance analysis revealed OTUs

144 that differed significantly between 0, 1, 4 and 9-minutes of bead-beating of mouse and human stool

145 (Supplementary Tables S5 \& S6). Supplementary Tables 7A\&B list genus level annotations of top

146 OTUs in mouse and human stool.

148 High bacterial diversity in bead beaten samples

149 Alpha diversity analysis showed higher phylogenetic richness in bead beaten samples as compared

150 to unbeaten samples (Supplementary Fig.2A, E). Shannon's entropy and Simpson's indices are

151 metrices that are commonly used for measurement of bacterial diversity. As shown in

152 Supplementary Fig. 2B \&F, higher Shannon entropy was observed after 1, 4 and 9-minutes of bead

153 beating as compared no bead beating. Similarly, Simpson's indices were higher in bead-beaten

154 samples, further suggesting high bacterial recovery at 4 and 9 minutes of bead beating

155 (Supplementary Fig. 2C\&G). As shown in Supplementary Tables S8 \& S9, bead beaten samples

156 showed a 1.1-fold increase in phylogenetic diversity, Simpson's index and Shannon entropy as

157 compared to unbeaten sample. Beta diversity analysis showed that all bead beaten samples

158 clustered more closely to one another than to unbeaten samples (Supplementary Fig. 2D\&H).

159 Overall, it was observed that most of the diversity was captured by beating for 4 minutes and no

160 significant increase in diversity was noticed with further bead beating. 


\section{Bead beating duration strongly impacts recovery of clinically relevant bacteria}

164 Differential abundance analysis on the most abundant OTUs revealed five clusters of bacteria (Fig.

165 3A, Supplementary Table S10). As shown, cluster 1 (C1) was comprised of Bifidobacterium and

166 Ruminicoccus in human stool. Maximum recovery of these bacteria was observed after 4 and 9

167 minutes of beating as compared to no bead beating ( $\mathrm{C} 1$ in Fig.3A). On the other hand, abundance

168 of Sutterella, Veillonella dispar and Veillonella parvula DNA was highest in samples that were

169 unbeaten or beaten for 1 minute as compared to samples beaten for 4 or 9 minutes (C2 in Fig. 3A).

170 Another cluster of bacteria in human stool was comprised of Blutia obeum, Bifidobacterium

171 longum, Coprococcus, Dorea and Streptococcus. These organisms were more highly represented

172 at the 4-minute timepoint and did not show a significant increase in recovery with longer bead

173 beating (i.e., 9 minutes). Cluster 4 (C4) was comprised of Lactobacillus reuteri, Allobaculum and

174 Bifidobacterium pseudolongum in mouse stool. Maximum abundance of these bacteria was

175 observed after 9 minutes of bead beating (Fig. 3A, C4). On the other hand, bacteria of the

176 Rikenellaceae, Desulfovibrio, Bacteroidales and Clostriadales groups showed maximum

177 abundance in unbeaten samples, as shown in cluster 5 (C5) of Fig. 3A.

179 Interestingly, we found that bead beating intensity has a strong impact on the recovery of clinically-

180 relevant inhabitants of mouse and human gut, including members of the genera Bifidobacterium,

181 Sutterella and Veillonella. As shown in Fig. 3B-E, replicates of mouse and human stool showed

182 maximum abundance of Bifidobacterium in samples beaten for 9 minutes, with 30 -100-fold higher

183 recovery in mouse and 2-16-fold higher recovery in human stool upon bead beating. On the other

184 hand, maximum abundance of Sutterella was observed in mouse and human stool samples that

185 were unbeaten or beaten for the least amount of time (Fig. 3F-I). We observed a 2-4-fold reduction 
186 in Sutterella abundance in bead beaten stool, suggesting an adverse effect of beating on recovery

187 of DNA from this bacterial group. These results were consistent across mouse and human stool

188 replicates (Fig. 3F-I, Supplementary data in Table S11-12).

\section{Optimum bead beating time for maximal recovery of microbiome diversity}

191 We compared various parameters including nucleic acid yield, DNA integrity, sequencing depth 192 and OTU counts across beating times in order to determine the optimum beating intensity for

193 mouse and human stool analysis. We found that optimum data were obtained with 4 and 9 minute

194 bead beating treatment as compared to no bead beating or beating for 1 minute. Comparison of 195 samples beaten for 4 and 9 minutes did not show marked differences. In data from mouse stool,

196 there were only 7 OTUs (out of 24 major OTUs) whose abundance differed significantly $(\mathrm{p}<0.05)$

197 between samples beaten for 4 and 9 minutes. These were Bifidobacterium, Adlercreutzia, 198 Allobaculum, Coriobacteriaceae, Lactobacillus, Turicibacter and Ruminicoccus (Supplementary 199 Table S7A-B). Similarly, Streptococcus, Suttrella, Dorea, Parabacteroides and Bifidobacterium

200 were 5 of 27 major OTUs in human stool that differed significantly $(\mathrm{p}<0.05)$ in samples beaten for 2014 versus 9 minutes. These results suggest that up to $70 \%$ of microbial signatures can be captured 202 with just 4 minutes of bead beating. However, stool samples rich in bacteria such as 203 Bifidobacterium, Streptococcus and Adlercreutzia, etc. may require more than 4 minutes of beating 204 for maximal DNA recovery. These results suggest that 4-5 minutes of bead beating may be 205 sufficient to capture most of the bacterial diversity in mouse and human stool. 


\section{Discussion:}

210 In this study we have systematically assessed the impact of bead beating on microbiome analysis

211 of mouse and human stool. Due to multiple technical and environmental factors, an accurate and

212 reproducible characterization of microbiota composition is a major challenge. Methods of sample

213 storage and collection, DNA extraction, sequencing library preparation and bioinformatics

214 analysis have been shown to contribute variability in $16 \mathrm{~S}$ results $(20-24)$. Of these, the DNA

215 extraction method is among the most important in that it can introduce bias at the initial step.

217 Several studies have reported optimization of DNA extraction methods and have developed

218 protocols for extracting microbial DNA from stool samples $(8,9)$. Large scale microbiome studies

219 such as Human Microbiome Project (HMP), MetaHIT, and the Earth Microbiome Project have

220 reported improved versions of DNA extraction protocols for various types of samples (25-27). The

221 published literature suggests that complete lysis of bacterial cell walls using beads can markedly

222 impact DNA yield as well downstream $16 \mathrm{~S}$ sequencing results $(28,29)$. Observed maximal

223 recovery of Actinobacteria in samples subjected to bead beating for 9 minutes is consistent with

224 published literature that reports enhanced nucleic acid recovery from Gram-positive organisms

225 with longer disruption of the bacterial cell wall (30). However, there are also other factors such as

226 volume and temperature of elution buffer, type of lysis beads, lysis tubes and columns that were

227 not evaluated in the current study but can also impact overall DNA yield and sequencing data 228 quality.

230 Our data suggest that bead beating duration strongly impacts the recovery of DNA from several 231 groups of bacteria. For example, optimization of the duration of bead beating enhanced DNA 
232 recovery from Bifidobacteria, Sutterella and Veillonella, three clinically-relevant bacterial groups

233 that are important members of the mouse and human gut microbiome (19, 31-35). Bifidobacterium,

234 a genus that is significantly underrepresented in the analysis of unbeaten stool, is one of the major

235 colonizers of the human gastrointestinal tract. These microbes have been shown to provide health

236 benefits to their host and are investigated in the context of various human diseases such as

237 colorectal cancer, necrotizing enterocolitis and inflammatory bowel diseases (31).

239 By contrast, we found that recovery of DNA from certain bacterial groups was reduced by bead 240 beating. For example, DNA from Sutterella and Veillonella showed reduced prevalence in 241 samples after bead beating, suggesting sensitivity of these microbes to extensive mechanical lysis.

242 These bacteria are also clinically relevant, as altered abundance of Sutterella has been associated 243 with many clinical conditions such as autism spectrum disorder, down syndrome and inflammatory

244 bowel disease $(32,33)$. Similarly, epidemiological studies in young children have associated 245 Veillonella with asthma (34), bronchiolitis (36) and autism (35). Since abundance of these 246 microbes could be clinically informative, it is important to be able accurately and precisely 247 determine their abundance in clinical specimens. Our data suggest that studies targeting 248 Bifidobacteria should incorporate longer (up to 9 minutes) bead beating protocols in order to 249 ensure maximal recovery of DNA from these bacteria, while those targeting organisms such as 250 Sutterella and Veillonella should avoid extensive bead beating for maximal recovery and accurate 251 representation. Our data indicate that 4-5 minutes of bead beating may be appropriate to process 252 samples where the composition of microbiomes are unknown. 
254 In summary, our study demonstrates that the duration of bead beating has a strong impact on the

255 recovery of DNA from clinically relevant microbiota in both mouse and human gut. Our data

256 suggest that a minimum of 4 minutes of bead beating (using Qiagen PowerLyzer) can result in

257 recovery of about $70 \%$ of gut microbiota DNA signatures. Further, our study identifies particular

258 groups of bacteria in mouse and human stool that can be recovered with up to 4 minutes of bead

259 beating and those that require extensive bead beating for maximal recovery. 
280 Acknowledgments:

281 This study was supported by the UT Southwestern Microbiome Research Laboratory. Authors

282 gratefully acknowledge donors of deidentified human stool samples for the study.

283

\section{Author contributions}

285 B.Z. and M.B. performed experiments; C.A. performed sequencing quality control, C.D. collected

286 mouse stool for study, L.V.H. contributed to manuscript editing and P.R. conceived and designed

287 the experiments and wrote the manuscript.

288

289 Competing interests: The authors declare no competing interests.

291 Data availability: Raw fastq files from mouse and human experiments have been deposited in

292 NCBI SRA database with accession no. PRJNA625828

293

294

295

296

297

298 
302 Figure legends.

\section{Figure 1. Experimental workflow for 16 S sequencing}

304 Illustration of the experimental workflow. Two mouse and two human stool samples were

305 homogenized using a PowerLyzer 24 Homogenizer (110/220V; Qiagen). DNA was extracted 306 using four different bead beating times, followed by 16S rRNA gene sequencing and analysis.

Figure 2. Actinobacteria are strongly impacted by bead beating in mouse and human stool

309 Panels A-B: Color coded bar plots showing the phylum level abundance across different bead

310 beating treatments in mouse and human stool, respectively. Panels C-D show abundance of

311 Actinobacteria across bead beating treatments in mouse and human stool, respectively. Panels

312 E-F show abundance of Firmicutes in mouse and human stool beaten for different times. Panel

313 G-H shows abundance of Bacteroidetes in mouse and human stool. Similarly, in Panels I-J,

314 bar plots show abundance of Proteobacteria across four bead beating time points in mouse

315 and human stool. Statistical p-values are denoted with *,\# and \$ represent comparison with

316 samples that were unbeaten, or beaten for 1 minute and 4 minutes, respectively.

317 Figure 3. Bacterial clusters defined by bead beating time

318 Panel A: Results of differential abundance analysis. The heatmap shows the top 30

319 differentially recovered OTUs in mouse and human stool. Panels B-E show the abundance of

320 Bifidobacterium across four beating treatments in mouse and human stool. Similarly, Panels

321 F-I show the abundance of Sutterella across four beating treatments in mouse and human

322 stool. Data from replicates of mouse and human sample is presented. Statistical p-values 
bioRxiv preprint doi: https://doi.org/10.1101/2020.06.15.151753; this version posted June 15, 2020. The copyright holder for this preprint (which was not certified by peer review) is the author/funder, who has granted bioRxiv a license to display the preprint in perpetuity. It is made available under aCC-BY 4.0 International license.

323 denoted with *, \# and \$ represent comparison with samples that were unbeaten, or beaten for

3241 minute and 4 minutes, respectively.

325

326

327

328

329

330

331

332

333

334

335

336

337

338

339

340

341

342

343

344

345 
347 References:

348 1. Werner JJ, Zhou D, Caporaso JG, Knight R, Angenent LT. Comparison of Illumina paired-end and

349 single-direction sequencing for microbial 16S rRNA gene amplicon surveys. The ISME journal.

$350 \quad 2012 ; 6(7): 1273-6$.

3512 2. The Integrative Human Microbiome Project. Nature. 2019;569(7758):641-8.

352 3. Gill SR, Pop M, Deboy RT, Eckburg PB, Turnbaugh PJ, Samuel BS, et al. Metagenomic analysis of

353 the human distal gut microbiome. Science. 2006;312(5778):1355-9.

354 4. Nelson KE, Weinstock GM, Highlander SK, Worley KC, Creasy HH, Wortman JR, et al. A catalog of 355 reference genomes from the human microbiome. Science. 2010;328(5981):994-9.

356 5. Bag S, Saha B, Mehta O, Anbumani D, Kumar N, Dayal M, et al. An Improved Method for High

357 Quality Metagenomics DNA Extraction from Human and Environmental Samples. Sci Rep. 2016;6:26775.

358 6. Baker GC, Smith JJ, Cowan DA. Review and re-analysis of domain-specific 16S primers. Journal of 359 microbiological methods. 2003;55(3):541-55.

360 7. Tremblay J, Singh K, Fern A, Kirton ES, He S, Woyke T, et al. Primer and platform effects on 16S

361 rRNA tag sequencing. Frontiers in microbiology. 2015;6:771.

362 8. Costea PI, Zeller G, Sunagawa S, Pelletier E, Alberti A, Levenez F, et al. Towards standards for

363 human fecal sample processing in metagenomic studies. Nat Biotechnol. 2017;35(11):1069-76.

364 9. Sinha R, Abu-Ali G, Vogtmann E, Fodor AA, Ren B, Amir A, et al. Assessment of variation in

365 microbial community amplicon sequencing by the Microbiome Quality Control (MBQC) project

366 consortium. Nat Biotechnol. 2017;35(11):1077-86.

367 10. Brooks JP, Edwards DJ, Harwich MD, Jr., Rivera MC, Fettweis JM, Serrano MG, et al. The truth 368 about metagenomics: quantifying and counteracting bias in 16S rRNA studies. BMC microbiology. $3692015 ; 15: 66$ 
370 11. Momozawa Y, Deffontaine V, Louis E, Medrano JF. Characterization of bacteria in biopsies of

371 colon and stools by high throughput sequencing of the V2 region of bacterial 16S rRNA gene in human.

372 PLoS One. 2011;6(2):e16952.

373 12. Willner D, Daly J, Whiley D, Grimwood K, Wainwright CE, Hugenholtz P. Comparison of DNA

374 extraction methods for microbial community profiling with an application to pediatric bronchoalveolar

375 lavage samples. PLoS One. 2012;7(4):e34605.

376 13. Wu GD, Lewis JD, Hoffmann C, Chen YY, Knight R, Bittinger K, et al. Sampling and

377 pyrosequencing methods for characterizing bacterial communities in the human gut using 165 sequence

378 tags. BMC microbiology. 2010;10:206.

379 14. Gill C, van de Wijgert JH, Blow F, Darby AC. Evaluation of Lysis Methods for the Extraction of

380 Bacterial DNA for Analysis of the Vaginal Microbiota. PLoS One. 2016;11(9):e0163148.

381 15. Valentini A, Taberlet P, Miaud C, Civade R, Herder J, Thomsen PF, et al. Next-generation

382 monitoring of aquatic biodiversity using environmental DNA metabarcoding. Mol Ecol. 2016;25(4):929-

38342.

384 16. von Wintzingerode F, Gobel UB, Stackebrandt E. Determination of microbial diversity in

385 environmental samples: pitfalls of PCR-based rRNA analysis. FEMS microbiology reviews.

$3861997 ; 21(3): 213-29$.

387 17. Dilhari A, Sampath A, Gunasekara C, Fernando N, Weerasekara D, Sissons C, et al. Evaluation of 388 the impact of six different DNA extraction methods for the representation of the microbial community 389 associated with human chronic wound infections using a gel-based DNA profiling method. AMB Express. $390 \quad 2017 ; 7(1): 179$.

391 18. Kim SJ, Chang J, Singh M. Peptidoglycan architecture of Gram-positive bacteria by solid-state 392 NMR. Biochim Biophys Acta. 2015;1848(1 Pt B):350-62. 
19. Fiedorova K, Radvansky M, Nemcova E, Grombirikova H, Bosak J, Cernochova M, et al. The

394 Impact of DNA Extraction Methods on Stool Bacterial and Fungal Microbiota Community Recovery. Front

395 Microbiol. 2019;10:821.

396 20. A review of 10 years of human microbiome research activities at the US National Institutes of

397 Health, Fiscal Years 2007-2016. Microbiome. 2019;7(1):31.

398 21. Penington JS, Penno MAS, Ngui KM, Ajami NJ, Roth-Schulze AJ, Wilcox SA, et al. Influence of

399 fecal collection conditions and 16S rRNA gene sequencing at two centers on human gut microbiota

$400 \quad$ analysis. Sci Rep. 2018;8(1):4386.

401 22. Carroll IM, Ringel-Kulka T, Siddle JP, Klaenhammer TR, Ringel Y. Characterization of the fecal

402 microbiota using high-throughput sequencing reveals a stable microbial community during storage. PLoS

403 One. 2012;7(10):e46953.

404 23. Gorzelak MA, Gill SK, Tasnim N, Ahmadi-Vand Z, Jay M, Gibson DL. Methods for Improving

405 Human Gut Microbiome Data by Reducing Variability through Sample Processing and Storage of Stool.

406 PLoS One. 2015;10(8):e0134802.

407 24. Rintala A, Pietila S, Munukka E, Eerola E, Pursiheimo JP, Laiho A, et al. Gut Microbiota Analysis

408 Results Are Highly Dependent on the 16S rRNA Gene Target Region, Whereas the Impact of DNA

409 Extraction Is Minor. J Biomol Tech. 2017;28(1):19-30.

$41025 . \quad$ Structure, function and diversity of the healthy human microbiome. Nature.

$411 \quad 2012 ; 486(7402): 207-14$.

412 26. Qin J, Li R, Raes J, Arumugam M, Burgdorf KS, Manichanh C, et al. A human gut microbial gene

413 catalogue established by metagenomic sequencing. Nature. 2010;464(7285):59-65.

414 27. Marotz C, Amir A, Humphrey G, Gaffney J, Gogul G, Knight R. DNA extraction for streamlined 415 metagenomics of diverse environmental samples. Biotechniques. 2017;62(6):290-3. 
416 28. Teng F, Darveekaran Nair SS, Zhu P, Li S, Huang S, Li X, et al. Impact of DNA extraction method

417 and targeted 16S-rRNA hypervariable region on oral microbiota profiling. Sci Rep. 2018;8(1):16321.

418 29. Lim MY, Song EJ, Kim SH, Lee J, Nam YD. Comparison of DNA extraction methods for human gut

419 microbial community profiling. Syst Appl Microbiol. 2018;41(2):151-7.

420 30. Yuan S, Cohen DB, Ravel J, Abdo Z, Forney LJ. Evaluation of methods for the extraction and 421 purification of DNA from the human microbiome. PLoS One. 2012;7(3):e33865.

422 31. O'Callaghan A, van Sinderen D. Bifidobacteria and Their Role as Members of the Human Gut

423 Microbiota. Front Microbiol. 2016;7:925.

424 32. Wang L, Christophersen CT, Sorich MJ, Gerber JP, Angley MT, Conlon MA. Increased abundance 425 of Sutterella spp. and Ruminococcus torques in feces of children with autism spectrum disorder. Mol

426 Autism. 2013;4(1):42.

427 33. Zhang M, Ma W, Zhang J, He Y, Wang J. Analysis of gut microbiota profiles and microbe-disease 428 associations in children with autism spectrum disorders in China. Sci Rep. 2018;8(1):13981.

429 34. Arrieta MC, Stiemsma LT, Dimitriu PA, Thorson L, Russell S, Yurist-Doutsch S, et al. Early infancy 430 microbial and metabolic alterations affect risk of childhood asthma. Sci Transl Med.

$431 \quad 2015 ; 7(307): 307 r a 152$.

432 35. Strati F, Cavalieri D, Albanese D, De Felice C, Donati C, Hayek J, et al. New evidences on the 433 altered gut microbiota in autism spectrum disorders. Microbiome. 2017;5(1):24.

434 36. Hasegawa K, Linnemann RW, Mansbach JM, Ajami NJ, Espinola JA, Petrosino JF, et al. The Fecal 435 Microbiota Profile and Bronchiolitis in Infants. Pediatrics. 2016;138(1). 


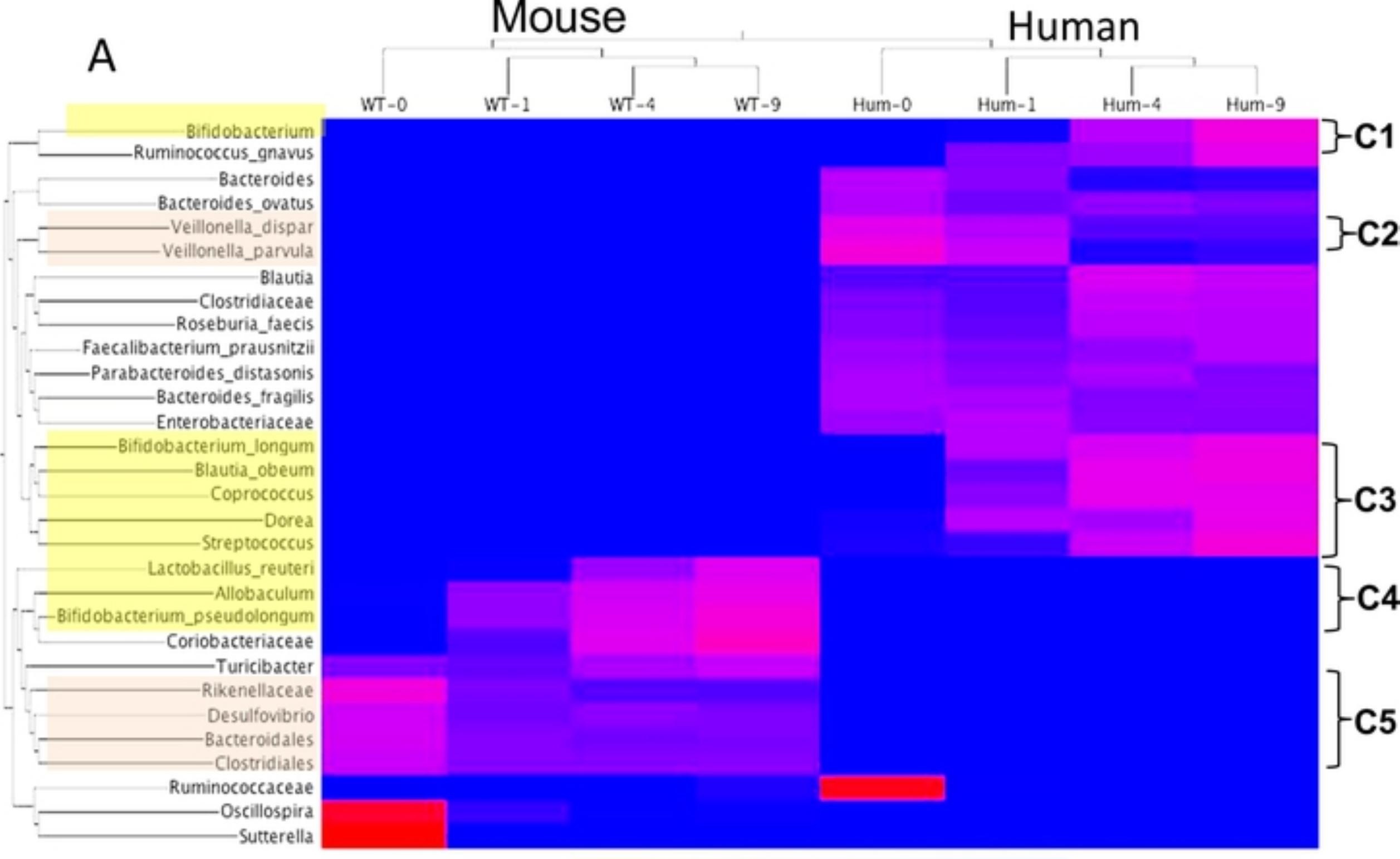

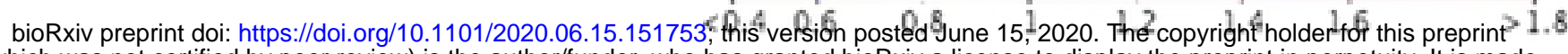
(which was not certified by peer review) is the author/funder, who has granted bioRxiv a license to display the preprint in perpetuity. It is made

B

Mouse-1: Bifidobacterium

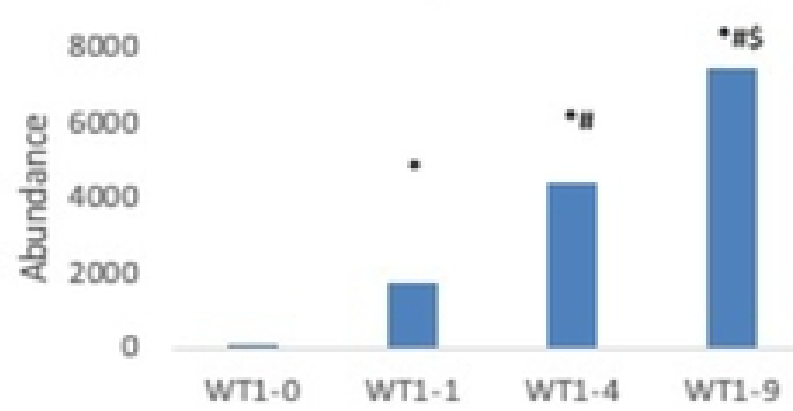

D Human-1: Bifidobacterium

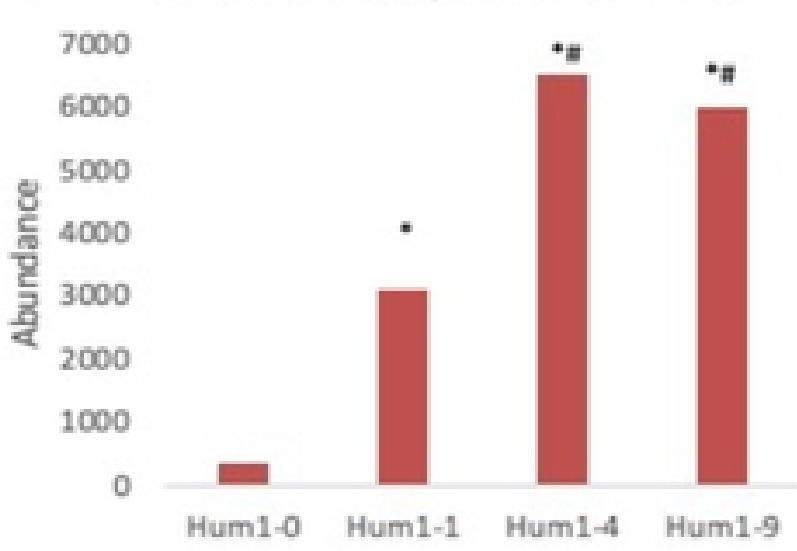

$\mathrm{F}$
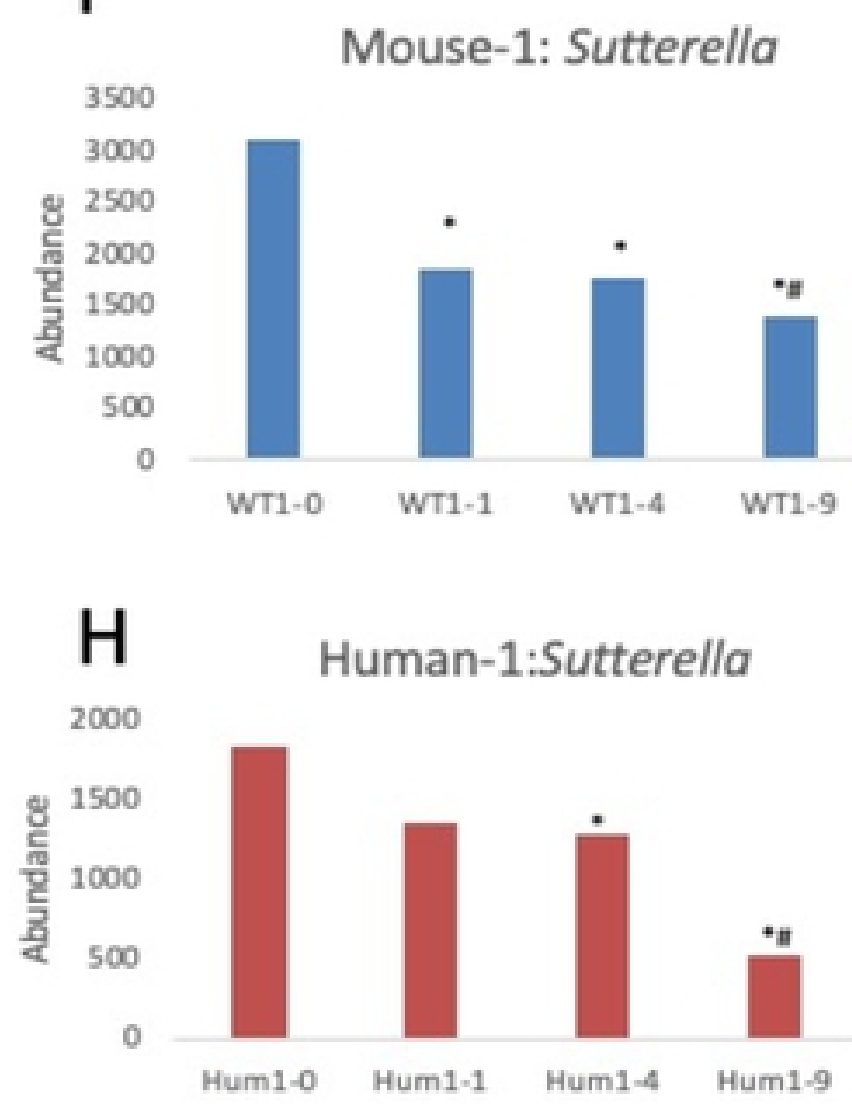

C

Mouse-2: Bifidobacterium

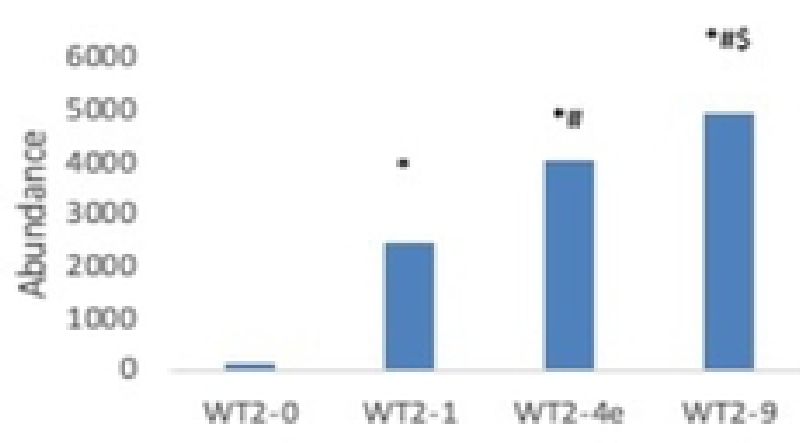

E Human-2: Bifidobacterium

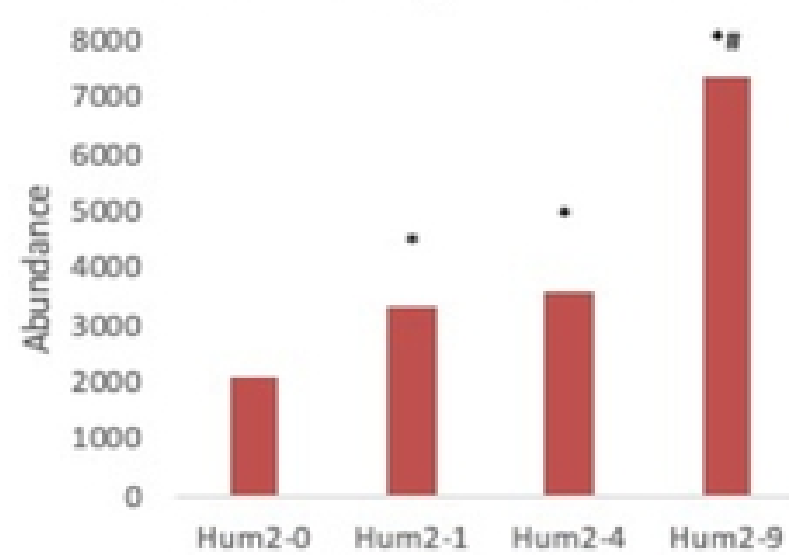

G
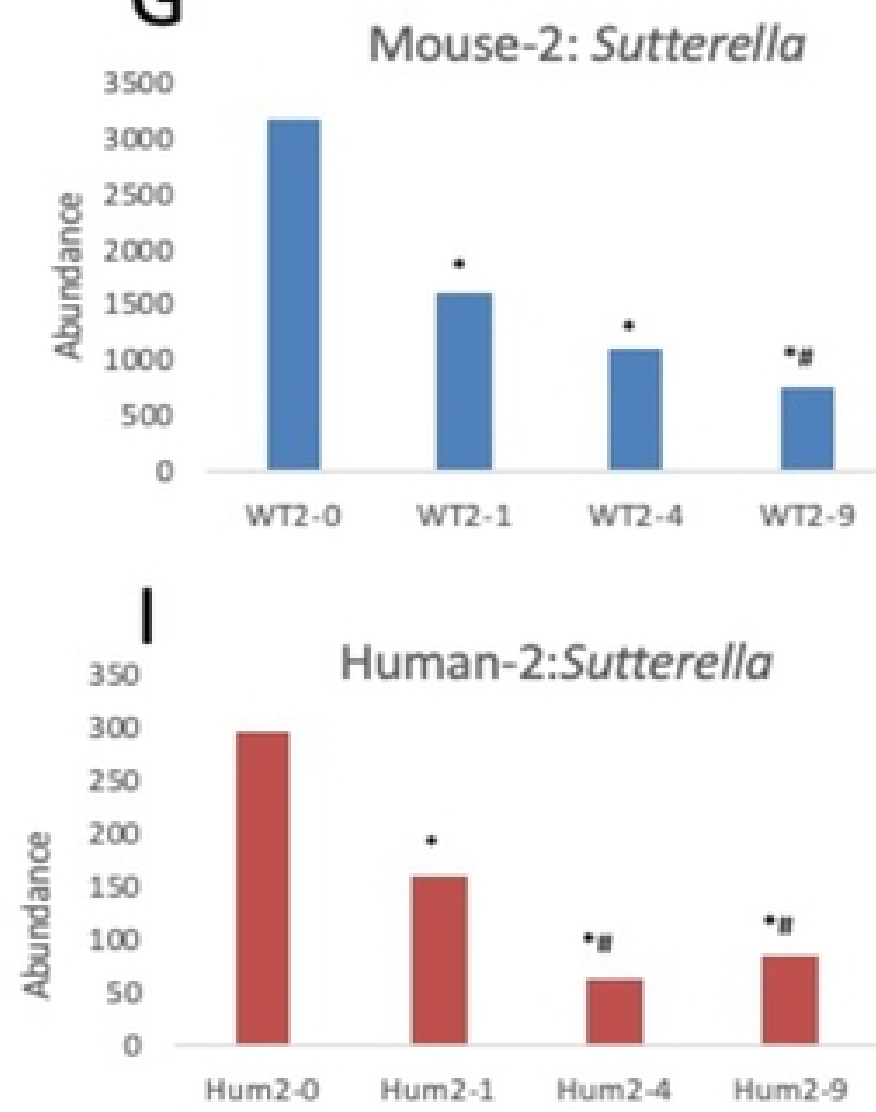

Fig 3 